\title{
Systemic Leukocyte Alterations in Cancer and their Relation to Prognosis
}

\author{
Beatriz Martins Tavares-Murta ${ }^{*}, 1$ and Eddie Fernando Candido Murta ${ }^{2}$ \\ ${ }^{I}$ Department of Biological Sciences, ${ }^{2}$ Oncological Research Institute (IPON)/Discipline of Gynecology and Obstetrics, \\ Federal University of Triângulo Mineiro, Uberaba-MG, Brazil
}

\begin{abstract}
Leukocyte migration is a key event in the inflammatory response to tumors. The tumor releases specific chemokines that control migration of leukocytes and functions of these cells after their arrival at the tumor site. In addition to these local changes in the tumor microenvironment, the host response to malignant solid tumors also gives rise to systemic effects, the most frequent of which are leukocytosis, neutrophilia and lymphopenia. These hematological findings are significantly correlated with advanced tumor stage and, therefore, poor disease prognosis. The ratio of neutrophil and lymphocyte counts has been suggested as a simple parameter of systemic inflammation in cancer patients. An elevated neutrophil to lymphocyte ratio has been shown to be an independent prognostic factor for cancers at various different sites, suggesting that this parameter is a clinically accessible and useful biomarker for patient survival. The effect of tumor development on circulating leukocyte number has not been clarified. One proposed mechanism is that tumor cells produce soluble factors such as granulocyte colony stimulating factor, which mobilize precursor cells in the bone marrow, or other mediators that alter cell differentiation. Leukocyte counts may be readily obtained at the time of diagnosis, and these data could be useful as stratification factors in clinical trials and in identifying patients with poor prognosis, leading to better treatment strategies.
\end{abstract}

Keywords: Leukocytosis, neutrophilia, lymphopenia, neutrophil to lymphocyte ratio, cancer.

\section{LEUKOCYTE MIGRATION}

Leukocyte migration is a key event in the inflammatory response. Polymorphonuclear neutrophils, a subset of leukocytes, are the first line of defense against microorganisms, and are promptly recruited to inflamed loci in response to infection or tissue injury. Circulating neutrophils migrate towards inflammatory mediators released at the injury site $[1,2]$.

Many classical chemotactic factors such as complement factor 5a (C5a), leukotriene $\mathrm{B}_{4}$, platelet-activating factor (PAF) and bacterial formyl-peptides (fMLP) are not specific for particular subsets of leukocytes [3]. However, chemokines, which are low molecular mass chemotactic cytokines, produced locally in many tissues, may selectively attract specific types of leukocytes. The chemokine family can be divided according to structure into C-X-C chemokines (e.g., IL-8), which mainly attract neutrophils, and $\mathrm{C}$-C chemokines (e.g., monocyte chemotactic protein (MCP)-1), which are chemotactic for a variety of leukocytes. Chemokine-mediated leukocyte activation stimulates the secretion of proteases that degrade the subendothelial extracellular matrix and facilitate leukocyte migration. Synchronization of the production and release of these chemoattractants to give appropriate circulating concentrations and precise timing of action is essential for all steps of leukocyte migration [4].

*Address correspondence to this author at the Department of Biological Sciences, Federal University of Triângulo Mineiro, Praça Manoel Terra, 330, 38015-050, Uberaba-MG, Brazil; Tel: (55) 34-3318-5467 / 5426; Fax: (55) 34-3318-5462; E-mail: bmurtafarmaco@dcb.uftm.edu.br
The migratory capacity of leukocytes is critical to their role as defense cells. Once activated, neutrophils are able to phagocytose, to release granular lytic enzymes and antimicrobial polypeptides into the phagolysosome, and to generate large amounts of reactive oxygen and nitrogen species, such as hydrogen peroxide $\left(\mathrm{H}_{2} \mathrm{O}_{2}\right)$ and nitric oxide (NO), which are key mediators of the microbicidal activity of leukocytes [1,5].

In patients with AIDS, antiretroviral drugs increase the neutrophil and monocyte migration activity and thereby reduce the incidence of infections [6]. In septic patients, a reduced neutrophil migration is observed compared to healthy volunteers, and migration is lowest in the group of non-survivor patients, suggesting that this dysfunction could contribute to the prognosis of the disease [7].

\section{LEUKOCYTE MIGRATION IN CANCER}

Infiltrating inflammatory cells play a role in the progression and spread of tumors $[2,8]$. By the release of specific chemokines, the tumor microenvironment controls leukocyte migration and other functions of these cells after their arrival at the tumor site. The autocrine production of chemokines by tumor cells attracts inflammatory cells such as monocytes and neutrophils and increases their survival, proliferation and dissemination [8]. The inflammatory component of a developing tumor may include a diverse leukocyte population including neutrophils, dendritic cells, macrophages, eosinophils, mast cells and lymphocytes, all of which are capable of producing a wide variety of mediators [9]. However, macrophages and lymphocytes are the most common cell types found infiltrated in the stroma and in neoplastic fluids $[10,11]$. 
These cells are attracted by specific chemokines such as MCP-1 and regulated upon activation, normal T-cell expressed, and secreted (RANTES). The relationship between elevated MCP-1 and RANTES expression and the presence of tumor-associated macrophages is evident in breast cancer [12] and in cervical cancer [13]. Clinical studies have demonstrated a correlation between the elevated number of infiltrated macrophages and poor prognosis for breast, prostate, ovarian, cervical, endometrial, esophageal and bladder cancers [14].

The infiltrating inflammatory cells have pleiotropic functions: inhibition of tumor growth by non-specific cytotoxic mechanisms and induction of cell lysis, or conversely promotion of tumor development through the release of inflammatory mediators [15]. Pro-inflammatory cytokines promote the migration, differentiation and activity of antigen-presenting cells and may favorably affect survival of cancer patients [13]. Tumor-infiltrating leukocytes can produce vascular endothelial growth factor (VEGF), IL-8 and matrix metalloproteinases that mediate tumor invasion and angiogenesis [16].

Also, prostaglandins and NO, produced by cyclooxygenase- 2 and inducible NO synthase respectively, influence human tumor development [17]. NO is consistently detected at nanomolar concentrations in the tumor microenvironment and has been found to promote tumorigenesis [18]. The factors that determine the outcome of these competing effects of the inflammatory response during tumor development are still not yet defined [15].

The host response to malignant tumors comprises not only local changes in tumor microenvironment, but also systemic effects. In terms of inflammatory response, neoplasia is a paradox. Tumors produce chemokines that recruit leukocytes, but a deficient inflammatory response is evident at sites remote from the tumor [8, 19]. Circulating chemokines could desensitize leukocytes to migratory stimuli or, alternatively, the tumor may produce antiinflammatory mediators. Therefore, a systemic deficiency in inflammatory response could coexist with continued leukocyte recruitment to the tumor site [8].

\section{ALTERATIONS IN LEUKOCYTE COUNTS IN CANCER PATIENTS}

The most frequent systemic alterations detected in patients with malignant solid tumors are leukocytosis and neutrophilia. These hematological conditions are significantly correlated with advanced disease and, consequently, with poor prognosis [20]. Leukocytosis is a condition often encountered in a clinical setting, usually caused by an increase in the number of neutrophils, which represent 50 to $60 \%$ of total leukocytes. Etiologically, leukocytosis is a primary pathological condition affecting the white blood cells, but it frequently arises as a reaction to infection, chronic inflammation and cancer [20].

Neutrophilia was found to be an independent prognostic factor, associated with reduced survival in human metastatic melanoma, pancreatic carcinoma, and renal carcinoma [16, $21,22]$. A high pretreatment count of neutrophils in blood was confirmed as an independent prognostic factor for short overall survival in stage IV melanoma patients undergoing interleukin-2 immunotherapy. Furthermore, a high overall leukocyte count was a predictor of short overall survival. Since immunotherapy with cytokines induces adverse effects in almost every patient, it is important to avoid treating patients who are predicted not to benefit from the treatment [23].

Baseline elevated leukocyte count in peripheral blood was associated with poor survival in patients with advanced non-small cell lung cancer treated with chemotherapy regimens consisting of cisplatin and gemcitabine. After performance status, leukocyte count was a strong prognostic factor of survival in non-small cell lung cancer patients [24].

An inverse association between lymphocyte count and cancer mortality was found in a study consisting of 8447 participants of both genders in a physical check-up program in Taiwan from 1995 to 1997. A lower lymphocyte count was associated with increased cancer mortality, especially from hepatoma [25]. In patients with advanced pancreatic carcinoma, the major immunological change found was the reduction of total lymphocytes in blood, and this was associated with disease progression [21]. It was reported that clinical improvement in cancer patients was associated with a lymphocyte count that exceeded $10 \%$ of total leukocytes [26].

The relationship between lymphocyte count and the clinical response to chemotherapy was evaluated in lung, colorectal, breast and prostate cancer patients. Independent of tumor histotype and chemotherapeutic regimen, the mean number of lymphocytes was significantly decreased in patients with progressive disease following chemotherapy. Conversely, lymphocytosis occurred in patients who achieved an objective tumor regression in response to chemotherapy, and lymphocyte count at the end of treatment was significantly higher than values seen before the onset of treatment. A possible mechanism for this increase in lymphocyte count may be modulation of the cytokine network by chemotherapy, that corrects elevated endogenous production of immunosuppressive cytokines [27]. In patients with locally advanced cervical carcinoma, univariate and multivariate analysis showed that a greater baseline lymphocyte count was the best predictor of a complete clinical response and progression-free survival after chemoradiation [28]. A study of patients with metastatic renal carcinoma during immunotherapy with interleukin-2 and interferon-alpha showed that changes from baseline in the total lymphocyte counts 4 weeks after treatment were significantly higher in the responding patients than in the non-responding patients, whereas no difference was observed in eosinophil counts [29].

An accumulation of immature or defective circulating leukocytes could facilitate a systemic immune dysfunction, while the presence of those cells at the tumor site could facilitate tumor growth. In several cancers - breast, prostate and glioma - it was found that systemic distribution of immature dendritic cells was more evident in patients with advanced disease [30]. Dendritic cells are antigen presenting-cells that are potent initiators of a primary immune response. They originate from bone marrow progenitors, which circulate in peripheral blood and subsequently give rise to immature dendritic cells that reside in peripheral tissues [31]. 


\section{ALTERATIONS IN THE NEUTROPHIL AND LYMPHOCYTE RATIO IN CANCER PATIENTS}

The ratio of neutrophil to lymphocyte counts (NLR) has been suggested as a simple measure of systemic inflammation and stress in critically ill cancer patients. In a prospective longitudinal observational study to investigate serial changes in circulating neutrophil and lymphocyte counts in sepsis following major unscheduled surgery it was shown that there is a correlation between the severity of clinical course and the grade of neutrophilia and lymphocytopenia. Thus, the NLR was proposed as an easily measurable parameter to be used routinely in intensive care units to indicate the severity of affliction [26].

In patients with early gastric cancer, e.g. - stage I prognosis correlated well with the NLR, but not with the total number of white blood cells, in that death was significantly more frequent among patients with an elevated NLR (>2) [32]. In patients with advanced gastric cancer an NLR >2.5 was considered an independent prognostic factor, providing a clinically accessible and useful biomarker for patient survival [33]. In a study of gender-related differences in the effect of gastrectomy on the NLR, females showed a significantly greater increase in the proportion of neutrophils compared to preoperative values, a larger decrease in lymphocytes and monocytes, and higher NLR values than males. These findings indicate that women showed more immune-compromised response to gastrectomy than men [34].

A univariate analysis of human colorectal cancer showed that patients with pre-operative NLR $\geq 5$ had reduced overall and cancer-specific survival compared to those with NLR $<5$ [35]. A prospective study evaluating the NLR on the first day after elective colorectal resection demonstrated that an NLR $>9.3$ was associated with an increased risk of complications, allowing targeted preventive measures [36]. In patients undergoing resection for colorectal liver metastasis, an elevated NLR ( $\geq 5)$ increased both the risk of death and the risk of recurrence: thus a preoperative NLR measurement may provide a simple method to identify patients with a poor prognosis. Univariate analysis showed elevated NLR to be the sole positive predictor of recurrence [37].

Recent data provide evidence for an association between elevated NLR and ovarian cancer. Preoperative NLR in epithelial ovarian cancer subjects was significantly higher than the NLR in subjects with benign ovarian tumors or benign gynecologic disease or in healthy controls. The sensitivity and specificity of NLR in detecting ovarian cancer was determined to be $66.1 \%$ and $82.7 \%$, respectively. Cox multivariate analysis showed that elevated NLR was the most powerful predictive indicator of an adverse outcome in ovarian cancer [38].

In a study of cervical cancer, alterations were detected in both the number and function of circulating neutrophils from patients with invasive cancer compared with patients at the pre- and micro-invasive stages and a control group consisting of healthy volunteers. A clear association was found between neutrophilia and advanced stage cervical cancer. An NLR >5 was present in most patients with advanced stage disease but in none of the pre-invasive stage patients [19]. An earlier study reported higher leukocyte counts in patients with cervical intraepithelial neoplasia grade 3 than in the control group [39]. This study and others show that elevated NLR in invasive cervical cancer is a consequence not only of neutrophilia but also of reduced lymphocyte count [40].

It remains to be established whether changes in leukocyte counts or in the neutrophil to lymphocyte ratio are a secondary phenomenon of prognostic relevance only, or reflect fundamental mechanistic events occurring in the endstages of tumor progression with implications for the survival of patients. Considering that some of these alterations are found in patients with early-stage cancer such as stage I gastric cancer [32] and grade 3 cervical intraepithelial neoplasia [39], a role for systemic leukocyte alterations in tumor progression cannot be ruled out.

\section{MECHANISMS INVOLVED IN LEUKOCYTE ALTERATIONS IN CANCER PATIENTS}

An intriguing question in cancer biology is how tumor development contributes to alterations in the number of circulating leukocytes (Fig. 1). One possible mechanism is the production of soluble factors such as granulocyte and macrophage colony stimulating factor (GM-CSF) by tumor cells, capable of mobilizing precursors in the bone marrow; or vascular endothelial growth factor (VEGF) and interleukin-6, both of which alter cell differentiation [30]. It was demonstrated that G-CSF and M-CSF are produced constitutively in murine mammary carcinoma cells, and that these factors act synergistically to stimulate granulocytes in vitro; this synergism may play a role in the marked granulocytosis observed in tumor-bearing animals [41].

In a 77-year-old patient with gastric cancer, immunohistochemical analysis demonstrated G-CSF expression in the advanced-stage, poorly differentiated adenocarcinoma, but not in the early-stage, well differentiated adenocarcinoma. The expression of G-CSF was accompanied by a change in leukocyte count from normal to marked leukocytosis by the advanced stage of disease [42]. Increased expression of G-CSF was found in a resected specimen from a patient with pancreatic cancer and the resulting increased serum level of G-CSF was accompanied by severe leukocytosis [43]. Primary pulmonary carcinoma was also reported to produce both G-CSF and parathyroid hormonerelated peptide, causing leukocytosis, hypercalcemia and IL6 production from the bone [44].

White blood cell count has been suggested as an indicator of disease activity to be closely monitored in patients with G-CSF-producing renal cell carcinoma. In one study, the production of G-CSF was revealed by positive immunohistochemical staining of the tumor tissue using anti-G-CSF antibody. It was observed that G-CSF contributed to leukocytosis, and both the serum G-CSF level and the white blood cell count correlated with tumor growth [45]. Marked leukocytosis, consisting primarily of mature granulocytes was also observed in a case of advanced stage G-CSFproducing squamous cell carcinoma of the cervix. After radiation therapy, white blood cell count returned to a normal level, accompanied by reduced serum levels of GCSF [46]. 


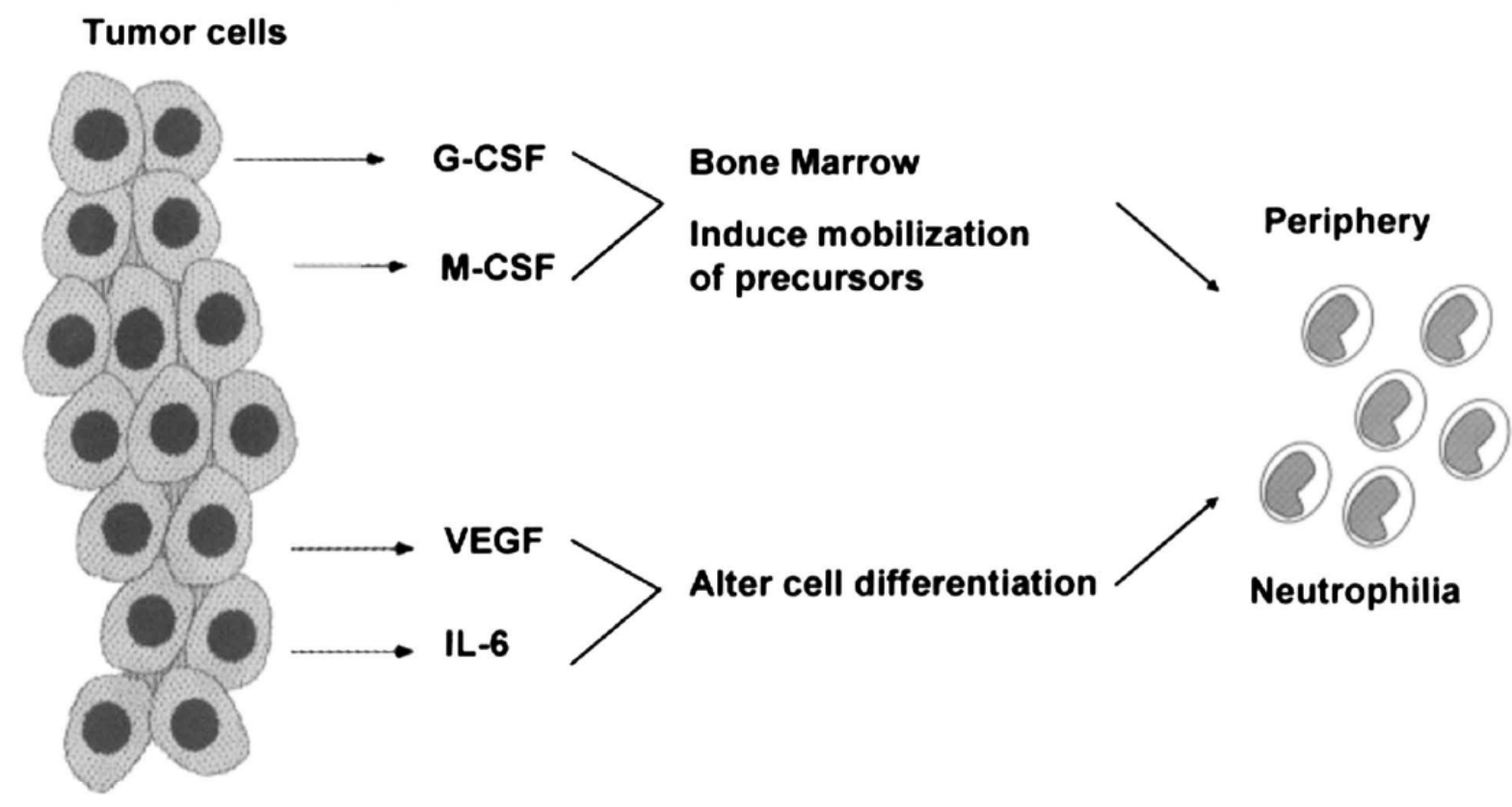

Fig. (1). Mechanisms involved in the induction of neutrophilia by tumor. G-CSF and M-CSF can function synergistically to stimulate the proliferation of granulocyte progenitors. G-CSF, granulocyte colony-stimulating factor; M-CSF, macrophage colony-stimulating factor; VEGF, vascular endothelial growth factor; IL-6, interleukin-6.

Pronounced neutrophilia is a manifestation of the strong host immune response elicited by photodynamic therapy of tumors. The cause of this neutrophilia was shown to be complement activation, which triggers the release of complement factors and at least a dozen secondary mediators including tumor necrosis factor- $\alpha$, IL-1 $\beta$, IL-6, IL-10, GCSF, prostaglandins and leukotrienes [47]. Another study showed that circulating neutrophils are persistently higher in mice deficient in the small GTPase Rac2 than in wild-type mice. Experiments using $\mathrm{Rac}^{-/}$and wild type cells suggested that Rac2 in hematopoietic cells regulates leukocyte lineage distribution and Rac2 in nonhematopoietic cells might contribute to regulating circulating neutrophil counts [48].

Lymphocyte depletion with consequent depression of innate cellular immunity is a severe clinical problem that can develop during cancer progression and cytoreductive therapies. Lymphopenia results from tumor-induced mechanisms that include impairment of antigen presentation, activation of negative costimulatory signals, and production of immunosuppressive factors, resulting in a marked decrease in T-helper lymphocytes [35, 49]. In addition, cancer cells may promote the expansion and recruitment of regulatory cell populations that contribute to the immunosuppressive network; these populations include regulatory $\mathrm{T}$ cells (Tregs), myeloid suppressor cells and distinct subsets of immature regulatory dendritic cells. Production of immunosuppressive mediators such as IL-10, NO and transforming growth factor- $\beta$ by tumor cells may also be responsible for evasion of immune surveillance; reduced production of these mediators restored a lymphoproliferative response [49]. Numerous strategies have been employed to induce a systemic anti-tumor immune response, including the adoptive transfer of cytolytic $\mathrm{T}$ cells and dendritic cell vaccination. However, these have demonstrated limited clinical success. Other investigators have induced the innate immune system to stimulate an anti-tumor response. The thymic pathway is commonly compromised in adults recovering from lymphopenia. As a result, $\mathrm{T}$ cells must rely upon peripheral expansion to restore cell numbers, through the stimulation of high cytokine levels [50].

\section{CONCLUDING REMARKS}

Patients with malignant solid tumors often develop paraneoplastic syndromes such as leukocytosis and neutrophilia, accompanied by an increased neutrophil to lymphocyte ratio. These conditions have proved to be useful predictors of response to treatment and survival, and have been proposed as independent prognostic factors. Thus these parameters could be useful as stratification factors in clinical trials and to identify patients with a poor prognosis prior to surgery. Results can be obtained from data already routinely available, without additional costs. Further understanding of the mechanisms giving rise to these conditions may contribute to the development of new therapeutic strategies in cancer and could benefit disease prognosis.

\section{ACKNOWLEDGEMENTS}

We thank Financiadora de Estudos e Projetos (FINEP), Fundação de Amparo à Pesquisa do Estado de Minas Gerais (FAPEMIG) and Conselho Nacional de Desenvolvimento Científico e Tecnológico (CNPq). We also thank Beatriz Cibele R. Gerolin for confection of figure.

\section{REFERENCES}

[1] Malawista SE, Montgomery RR, van Blaricom G. Evidence for reactive nitrogen intermediates in killing of staphylococci by human neutrophil cytoplasts. A new microbicidal pathway for polymorphonuclear leukocytes. J Clin Invest 1992; 90: 631-6.

[2] Coussens LM, Werb Z. Inflammation and cancer. Nature 2002; 420: 860-7. 
[3] Bokoch GM. Chemoattractant signaling and leukocyte activation. Blood 1995; 86: 1649-60.

[4] Proost P, Wuyts A, Van Damme J. The role of chemokines in inflammation. Int J Clin Lab Res 1996; 26: 211-23.

[5] Fierro IM, Nascimento-DaSilva V, Arruda MA, et al. Induction of NOS in rat blood PMN: in vivo and in vitro modulation by tyrosine kinase and involvement in bactericidal activity. J Leukoc Biol 1999; 65: 508-14.

[6] Mastroianni CM, Lichtner M, Mengoni F, et al. Improvement in neutrophil and monocyte function during highly active antiretroviral treatment of HIV-1-infected patients. AIDS 1999; 13: 883-90.

[7] Tavares-Murta BM, Zaparoli M, Ferreira RB, et al. Failure of neutrophil chemotactic function in septic patients. Crit Care Med 2002; 30: 1056-61.

[8] Balkwill F, Mantovani A. Inflammation and cancer: back to Virchow? Lancet 2001; 357: 539-45.

[9] Wahl LM, Kleinman HK. Tumor-associated macrophages as targets for cancer therapy. J Natl Cancer Inst 1998; 90: 1583-4.

[10] Mantovani G, Macciò A, Pisano M, et al. Tumor-associated lympho-monocytes from neoplastic effusions are immunologically defective in comparison with patient autologus PBMCs but are capable of releasing high amounts of various cytokines. Int $\mathbf{J}$ Cancer 1997; 71: 724-31.

[11] Tavares-Murta BM, Cunha FQ, Miranda R, Adad S, Murta EFC. Differential tumor microenvironment in human ovarian cystic tumors. Tumori 2004; 90: 491-7.

[12] Ben-Baruch A. Inflammation-associated immune suppression in cancer: The roles played by cytokines, chemokines and additional mediators. Sem Cancer Biol 2006; 16: 38-52.

[13] Zijlmans HJ, Fleuren GJ, Baelde HJ, Eilers PH, Kenter GG, Gorter A. Role of tumor-derived proinflammatory cytokines GM-CSF, TNF-alpha, and IL-12 in the migration and differentiation of antigen-presenting cells in cervical carcinoma. Cancer 2007; 109: 556-65.

[14] Shih J-Y, Yuan A, Chen JJ-W, Yang P-C. Tumor-associated macrophage: Its role in cancer invasion and metastasis. J Cancer Mol 2006; 2: 101-6.

[15] Al-Sarireh B, Eremin O. Tumor-associated macrophages (TAMs): disordered function, immune suppression and progressive tumor growth. J R Coll Sur Edinb 2000; 45: 1-16.

[16] Schmidt H, Bastholt L, Geertsen P, et al. Elevated neutrophil and monocyte counts in peripheral blood are associated with poor survival in patients with metastatic melanoma: a prognostic model. Br J Cancer 2005; 93: 273-8.

[17] Kong G, Kim EK, Kim WS, et al. Role of cyclooxygenase-2 and inducible nitric oxide synthase in pancreatic cancer. J Gastroenterol Hepatol 2002; 17: 914-21.

[18] Pervin S, Singh R, Hernandez E, Wu G, Chaudhuri G. Nitric oxide in physiologic concentrations targets the translational machinery to increase the proliferation of human breast cancer cells: involvement of mammalian target of rapamycin/eIF4E pathway. Cancer Res 2007; 67: 289-99.

[19] Fernandes Jr PC, Garcia CB, Micheli DC, Cunha FQ, Murta EFC, Tavares-Murta BM. Circulating neutrophils may play a role in the host response in cervical cancer. Int J Gynecol Cancer 2007; 17: 1068-74.

[20] Ruka W, Rutkowski P, Kaminska J, Rysinska A, Steffen J. Alterations of routine blood tests in adult patients with soft tissue sarcomas: Relationships to cytokine serum levels and prognostic significance. Ann Oncol 2001; 12: 1423-32.

[21] Fogar P, Sperti C, Basso D, et al. Decreased total lymphocyte counts in pancreatic cancer: An index of adverse outcome. Pancreas 2006; 32: 22-8.

[22] Donskov F, von der Maase H. Impact of immune parameters on long-term survival in metastatic renal cell carcinoma. J Clin Oncol 2006; 24: 1997-2005.

[23] Schmidt H, Suciu S, Punt CJ, et al. Pretreatment levels of peripheral neutrophils and leukocytes as independent predictors of overall survival in patients with American Joint Committee on Cancer Stage IV Melanoma: results of the EORTC 18951 Biochemotherapy Trial. J Clin Oncol 2007; 25: 1562-9.

[24] Tibaldi C, Vasile E, Bernardini I, Orlandini C, Andreuccetti M, Falcone A. Baseline elevated leukocyte count in peripheral blood is associated with poor survival in patients with advanced non-small cell lung cancer: a prognostic model. J Cancer Res Clin Oncol 2008; 134: 1143-9.

[25] Huang ZS, Chien KL, Yang CY, Wang CH, Chang TC, Chen CJ. Peripheral differential leukocyte counts and subsequent mortality from all diseases, cancers, and cardiovascular diseases in Taiwanese. J Formos Med Assoc 2003; 102: 775-81.

[26] Zahorec R. Ratio of neutrophil to lymphocyte counts - rapid and simple parameter of systemic inflammation and stress in critically ill. Bratisl Lek Listy 2001; 102: 5-14.

[27] Lissoni P, Fumagalli L, Brivio F, et al. Cancer chemotherapyinduced lymphocytosis: a revolutionary discovery in the medical oncology. J Biol Regul Homeost Agents 2006; 20: 29-35.

[28] Choi CH, Kang H, Him WY, et al. Prognostic value of baseline lymphocyte count in cervical carcinoma treated with concurrent chemoradiation. Int J Radiat Oncol Biol Phys 2008; 71: 199-204.

[29] Jeong IG, Han KS, Joung JY, et al. Analysis of changes in the total lymphocyte and eosinophil count during immunotherapy for metastatic renal cell carcinoma: correlation with response and survival. J Korean Med Sci 2007; 22: 122-8.

[30] Pinzon-Charry A, Ho CSK, Laherty R, et al. A Population of HLA$\mathrm{DR}^{+}$immature cells accumulates in the blood dendritic cell compartment of patients with different types of cancer. Neoplasia 2005; 7: 1112-22.

[31] Kah-Wai L, Jacek T, Jacek R. Dendritic cells heterogeneity and its role in cancer immunity. J Cancer Res Ther 2006; 2: 35-40.

[32] Hirashima M, Higuchi S, Sakamoto K, Nishiyama T, Okada H. The ratio of neutrophils to lymphocytes and the phenotypes of neutrophils in patients with early gastric cancer. J Cancer Res Clin Oncol 1998; 124: 329-34.

[33] Yamanaka T, Matsumoto S, Teramukai S, Ishiwata R, Nagai Y, Fukushima M. The baseline ratio of neutrophils to lymphocytes is associated with patient prognosis in advanced gastric cancer. Oncology 2007; 73: 215-20.

[34] Gwak MS, Choi SJ, Kim JA, et al. Effects of gender on white blood cell populations and neutrophil-lymphocyte ratio following gastrectomy in patients with stomach cancer. J Korean Med Sci 2007; 22: 104-8

[35] Walsh SR, Cook EJ, Goulder F, Justin TA, Keeling NJ. NeutrophilLymphocyte ratio as a prognostic factor in colorectal cancer. J Surg Oncol 2005; 91: 181-4.

[36] Cook EJ, Walsh SR, Farooq N, Alberts JC, Justin TA, Keeling NJ. Post-operative neutrophil-lymphocyte ratio predicts complications following colorectal surgery. Int J Surg 2007; 5: 27-30.

[37] Halazun KJ, Aldoori A, Malik HZ, et al. Elevated preoperative neutrophil to lymphocyte ratio predicts survival following hepatic resection for colorectal liver metastasis. Eur J Surg Oncol 2008; 34: 55-60.

[38] Cho H, Hur HW, Kim SW, et al. Pre-treatment neutrophil to lymphocyte ratio is elevated in epithelial ovarian cancer and predicts survival after treatment. Cancer Immunol Immunother 2009; 58: 15-23.

[39] Bais AG, Beckmann I, Lindemans J, et al. A shift to a peripheral Th2-type cytokine pattern during the carcinogenesis of cervical cancer becomes manifest in CIN III lesions. J Clin Pathol 2005; 58: 1096-100.

[40] García-González JE, Rojas-Espinosa O, Aguilar-Santelises M. Phagocytic activity of circulating polymorphonuclear leukocytes from patients with carcinoma of the uterine cervix. Rev Latinoam Microbiol 1992; 34: 135-41.

[41] Lee MY, Kaushansky K, Judkins SA, Lottsfeldt JL, Waheed A, Shadduck RK. Mechanisms of tumor-induced neutrophilia: constitutive production of colony-stimulating factors and their synergistic actions. Blood 1989; 74: 115-22.

[42] Yamano T, Morii E, Ikeda J, Aozasa K. Granulocyte colonystimulating factor production and rapid progression of gastric cancer after histological change in the tumor. Jpn J Clin Oncol 2007; 37: 793-6.

[43] Takami K, Miura K, Takeuchi H, et al. Granulocyte colonystimulating factor-producing pancreatic cancer: Report of a case. Surg Today 2008; 38: 453-7.

[44] Takaoka S, Yamane Y, Nishiki M, Yamaguchi T, Sugimoto T. Primary pulmonary squamous cell carcinoma associated with elevated IL-6, leukocytosis, hypercalcemia, phagocytosis, reactive lymphadenopathy and glomerular mesangial cell proliferation via the production of PTH-rP and G-CSF. Intern Med 2008; 47: 275-9. 
[45] Wang YC, Yang S, Tzen CY, Lin CC, Lin J. Renal cell carcinoma producing granulocyte colony-stimulating factor. J Formos Med Assoc 2006; 105: 414-7.

[46] Nasu K, Inoue C, Takai N, Kashima K, Miyakawa I. Squamous cell carcinoma of the cervix producing granulocyte colony-stimulating factor. Obstet Gynecol 2004; 104: 1086-8.

[47] Cecic I, Korbelik M. Mediators of peripheral blood neutrophilia induced by photodynamic therapy of solid tumors. Cancer Lett 2002; 183: 43-51.

[48] Gomez JC, Soltys J, Okano K, Dinauer MC, Doerschuk CM. The role of Rac2 in regulating neutrophil production in the bone marrow and circulating neutrophil counts. Am J Pathol 2008; 173: 507-17.

[49] Croci DO, Fluck MFZ, Rico MJ, Matar P, Rabinovich GA, Scharovsky OG. Dynamic cross-talk between tumor and immune cells in orchestrating the immunosuppressive network at the tumor microenvironment. Cancer Immunol Immunother 2007; 56: 16871700 .

[50] Williams KM, Hakim FT, Gress RE. T cell immune reconstitution following lymphodepletion. Semin Immunol 2007; 19: 318-30.

(C) Tavares-Murta and Murta; Licensee Bentham Open.

This is an open access article licensed under the terms of the Creative Commons Attribution Non-Commercial License (http: //creativecommons.org/licenses/by$\underline{\mathrm{nc} / 3.0 /)}$, which permits unrestricted, non-commercial use, distribution and reproduction in any medium, provided the work is properly cited. 